University of Nebraska - Lincoln

DigitalCommons@University of Nebraska - Lincoln

3-23-2005

\title{
Nanoelectromechanics of polarization switching in piezoresponse force microscopy
}

\author{
Sergei V. Kalinin \\ Oak Ridge National Laboratory, sergei2@ornl.gov \\ Alexei Gruverman \\ University of Nebraska-Lincoln, agruverman2@unl.edu \\ B. J. Rodriguez \\ North Carolina State University, Raleigh, brian.rodriguez@ucd.ie \\ J. Shin \\ Condensed Matter Sciences Division, Oak Ridge National Laboratory, Oak Ridge, Tennessee \\ E. Karapetian \\ Suffolk University, Boston, Massachusetts \\ See next page for additional authors
}

Follow this and additional works at: https://digitalcommons.unl.edu/physicsgruverman

Part of the Physics Commons

Kalinin, Sergei V.; Gruverman, Alexei; Rodriguez, B. J.; Shin, J.; Karapetian, E.; and Kachanov, M., "Nanoelectromechanics of polarization switching in piezoresponse force microscopy" (2005). Alexei Gruverman Publications. 29.

https://digitalcommons.unl.edu/physicsgruverman/29

This Article is brought to you for free and open access by the Research Papers in Physics and Astronomy at DigitalCommons@University of Nebraska - Lincoln. It has been accepted for inclusion in Alexei Gruverman Publications by an authorized administrator of DigitalCommons@University of Nebraska - Lincoln. 


\section{Authors}

Sergei V. Kalinin, Alexei Gruverman, B. J. Rodriguez, J. Shin, E. Karapetian, and M. Kachanov 


\title{
Nanoelectromechanics of polarization switching in piezoresponse force microscopy
}

\author{
S. V. Kalinin ${ }^{\text {a) }}$ \\ Condensed Matter Sciences Division, Oak Ridge National Laboratory, Oak Ridge, Tennessee 37831
}

A. Gruverman and B. J. Rodriguez

Department of Materials Science and Engineering, North Carolina State University, Raleigh, North Carolina 27695

J. Shin and A. P. Baddorf

Condensed Matter Sciences Division, Oak Ridge National Laboratory, Oak Ridge, Tennessee 37831

E. Karapetian

Department of Mathematics and Computer Science, Suffolk University, Boston, Massachusetts 02108

M. Kachanov

Department of Mechanical Engineering, Tufts University, Medford, Massachusetts 02155

(Received 4 October 2004; accepted 10 January 2005; published online 23 March 2005)

\begin{abstract}
Nanoscale polarization switching in ferroelectric materials by piezoresponse force microscopy in weak and strong indentation limits is analyzed using exact solutions for coupled electroelastic fields under the tip. Tip-induced domain switching is mapped on the Landau theory of phase transitions, with domain size as an order parameter. For a point charge interacting with a ferroelectric surface, switching by both first and the second order processes is possible, depending on the charge-surface separation. For a realistic tip, the domain nucleation process is first order in charge magnitude and polarization switching occurs only above a certain critical tip bias. In pure ferroelectric or ferroelastic switching, the late stages of the switching process can be described using a point charge model and arbitrarily large domains can be created. However, description of domain nucleation and the early stages of growth process when the domain size is comparable with the tip curvature radius (weak indentation) or the contact radius (strong indentation) requires the exact field structure. For higher order ferroic switching (e.g., ferroelectroelastic), the domain size is limited by the tip-sample contact area, thus allowing precise control of domain size. (c) 2005 American Institute of Physics.

[DOI: $10.1063 / 1.1866483$ ]
\end{abstract}

\section{INTRODUCTION}

The drive towards nanotechnology necessitates the development of ways to control properties of matter at the nanoscale. In the last several years, significant attention has been devoted to the applications of piezoresponse force microscopy (PFM) to the characterization of ferroelectric materials, which are used in high-density nonvolatile memories and other electronic devices. ${ }^{1-3}$ PFM provides an approach to nanoscale engineering via local modification and control of ferroelectric domain structures, with $\sim 10-30 \mathrm{~nm}$ resolution. ${ }^{4-8}$ The practical viability of these PFM applications depends on the minimal stable domain size that can be formed during local polarization switching induced by a tipgenerated field. Analysis of domain switching processes using a point charge approximation and the Landauer model for domain geometry was given by Molotskii et al. ${ }^{9,10}$ and, independently, by Abplanalp. ${ }^{11}$ Here we illustrate that the point charge model is valid only if the domain size is larger than the tip size, limiting its applicability to the late stages of the switching process. The stages of the polarization switching process, such as domain nucleation and early stages of

\footnotetext{
a) Author to whom correspondence should be addressed; electronic mail: sergei2@ornl.gov
}

growth, which are most relevant to applications in highdensity ferroelectric data storage devices, cannot be described using a point charge approximation. Moreover, even though it has been shown that strains produced by the tip may suppress local polarization ${ }^{12}$ or induce local ferroelectroelastic polarization switching, electrostatic models do not take into account strain effects. ${ }^{13,14}$ Here, we analyze the microscopic mechanisms for electric field- and mechanical stress-induced polarization switching phenomena using recently obtained expressions for the electroelastic fields under the PFM tip, ${ }^{15}$ for strong and weak indentation regimes. ${ }^{16}$

\section{THEORY}

Polarization switching in ferroelectric materials is a multiple step process that includes initial domain nucleation and subsequent forward and lateral domain expansion. ${ }^{17,18}$ Most ferroelectric materials are characterized by extremely thin domain walls and high wall energies, resulting in high activation barriers for a homogeneous domain nucleation in a uniform field. ${ }^{19}$ Once the critical nucleus size is achieved, the domains grow indefinitely, resulting in macroscopic switching in the crystal. The free energy evolution in this process is illustrated in Fig. 1(a). This simple picture no longer holds for PFM tip-induced switching. In this case, the 

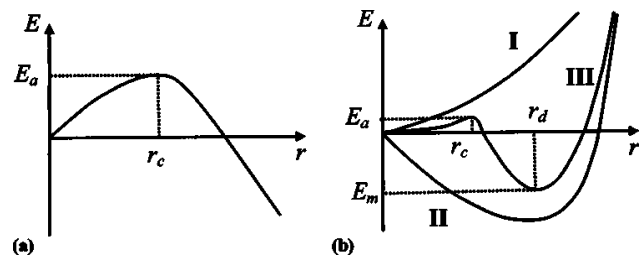

FIG. 1. (a) The domain free energy as a function of lateral domain size for a uniform field. (b) Possible scenarios for the domain free energy evolution for tip-induced switching. Case I: switching doesn't occur (external field is below the threshold value). Case II: nonactivation type of switching (external field is arbitrarily large); domain nucleates directly below the tip. Case III: activation type of switching (external field is above the threshold value); domain nucleation requires overcoming the activation barrier $E_{a}$.

electroelastic fields established by the tip are confined to the finite volume of the material, implying that tip-induced ferroelectric switching will be spatially limited and imposing the upper thermodynamic limit on the switched domain size. However, the early stages of the switching process, i.e., domain nucleation and lateral domain growth, can be expected to be similar to the uniform field case.

Simple estimates suggest that the electrical field under the tip $\left(>10^{7} \mathrm{~V} / \mathrm{m}\right)$ can be significantly larger than those achieved in macroscopic experiments. Hence, the activation energy and the critical size for domain nucleation may be sufficiently small to allow homogeneous nucleation under the tip. The three qualitatively different polarization switching scenarios in a PFM experiment are sketched in Fig. 1(b). Scenario I corresponds to the situation when a tip-induced field is insufficient to induce polarization switching below the tip. Scenario II corresponds to the case when the activation energy for domain nucleation is zero and a domain forms instantly. Finally, Scenario III corresponds to the case when domain nucleation requires a nonzero activation barrier to be overcome. In cases II and III, the final size of the domain is limited by the spatial extent of the electroelastic fields produced by the tip.

Here we analyze the thermodynamics of the scanning probe microscopy induced switching process including domain nucleation and final domain shape, using exact expressions for the electroelastic fields under the PFM tip in the weak and strong indentation regimes. ${ }^{15}$ It is shown that the tip-induced polarization switching can be described in the framework of the Landau theory of phase transitions, with the domain size as an order parameter.

The driving force for the $180^{\circ}$ polarization switching process in ferroelectrics is the change in the bulk free energy density: ${ }^{11,13}$

$$
\Delta g_{\text {bulk }}=-\Delta P_{i} E_{i}-\Delta d_{i \mu} E_{i} X_{\mu}
$$

where $P_{i}, E_{i}, X_{\mu}$, and $d_{i \mu}$, are components of the polarization, electric field, stress, and piezoelectric constants tensor, $i$ $=1,2,3$, and $\mu=1, \ldots, 6$. The first and second terms in Eq. (1) describe ferroelectric and ferroelectroelastic switching, respectively. Notably, for ferroelectric materials such as $\mathrm{LiNbO}_{3}$ and lead-zirconate-titanate (PZT), the signs of the corresponding free energy terms are opposite and the polarities of the domains formed by ferroelectric and ferroelectro-

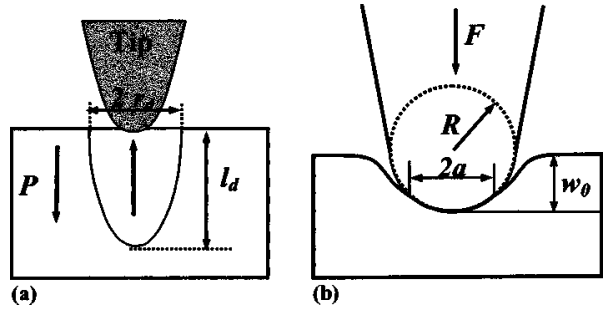

FIG. 2. (a) The domain geometry during tip-induced switching and (b) geometric parameters of the tip-surface junction.

elastic switching are reverse, thus providing an approach to distinguish these switching mechanisms.

The free energy of the nucleating domain is

$$
\Delta G=\Delta G_{\text {bulk }}+\Delta G_{\text {wall }}+\Delta G_{\text {dep }},
$$

where the first term is the change in bulk free energy, $\Delta G_{\text {bulk }}=\int \Delta g_{\text {bulk }} d V$, the second term is the domain wall energy, and the third term is the depolarization field energy. In the Landauer model of switching, the domain shape is approximated as a half ellipsoid with the small and large axis equal to $r_{d}$ and $l_{d}$, correspondingly [Fig. 2(a)]. The domain wall contribution to the free energy in this geometry is approximated as $\Delta G_{\text {wall }}=b r_{d} l_{d}$, where $b=\sigma_{\text {wall }} \pi^{2} / 2$ and $\sigma_{\text {wall }}$ is the direction-independent domain wall energy. The depolarization energy contribution is $\Delta G_{\mathrm{dep}}=c r_{d}^{4} / l_{d}$, where

$$
c=\frac{4 \pi P_{s}^{2}}{3 \varepsilon_{11}}\left[\ln \left(\frac{2 l_{d}}{r_{d}} \sqrt{\frac{\varepsilon_{11}}{\varepsilon_{33}}}\right)-1\right]
$$

has only a weak dependence on the domain geometry. ${ }^{20}$

The mechanism of polarization switching can be analyzed using free energy surfaces representing the domain energy as a function of $l_{d}, r_{d}$. A free energy surface calculated for $\mathrm{BaTiO}_{3} \quad\left(\sigma=7 \mathrm{~mJ} / \mathrm{m}^{2}, P_{s}=0.26 \mathrm{C} / \mathrm{m}^{2}, \varepsilon_{11}=2000, \varepsilon_{33}\right.$ $=120)^{21}$ for the uniform field $E=10^{5} \mathrm{~V} / \mathrm{m}$ is shown in Fig. 3(a). The free energy surface has a saddle point and the domain grows indefinitely once the critical size corresponding to activation barrier for nucleation $E_{a}$ is reached [compare with Fig. 1(a)]. Minimization of Eq. (2) with respect to $r_{d}$ and $l_{d}$ allows one to estimate the critical domain size and activation energy for nucleation, $r_{c}=5 \mathrm{~b} / 6 a, l_{c}$ $=5^{3 / 2} b c^{1 / 2} / 6 a^{3 / 2}$, and $E_{a}=5^{5 / 2} b^{3} c^{1 / 2} / 108 a^{5 / 2}$, where $a$ $=4 \pi P_{s} E / 3$. For the parameters in the text, the activation energy for domain nucleation is $E_{a}=2.4 \times 10^{5} \mathrm{eV}$ for $l_{c}$ $=16.4 \mu \mathrm{m}, r_{c}=0.264 \mu \mathrm{m}$. Thus, for relatively weak fields corresponding to experimental coercive fields, homogeneous domain nucleation is impossible and in typical ferroelectric materials domain nucleation occurs on the surface or at interface defects. However, the activation energy for nucleation is a strong function of the field, and for the fields on the order of $10^{7} \mathrm{~V} / \mathrm{m}$ generated by the tip of radius $\sim 100 \mathrm{~nm}$ at potential of $1 \mathrm{~V}$, the corresponding parameters are: $E_{a}$ $=2.17 \mathrm{eV}$ for $l_{c}=11.4 \mathrm{~nm}, r_{c}=2.6 \mathrm{~nm}$. The strong scaling of $E_{a}$ with bias suggests that even for relatively low tip biases on the order of $1-10 \mathrm{~V}$, the activation energy is small enough to allow thermal fluctuations to overcome the activation barrier, resulting in ferroelectric domain formation under the tip. Thus, domain nucleation in PFM does not require an impurity or other nucleation center as in the uniform field 

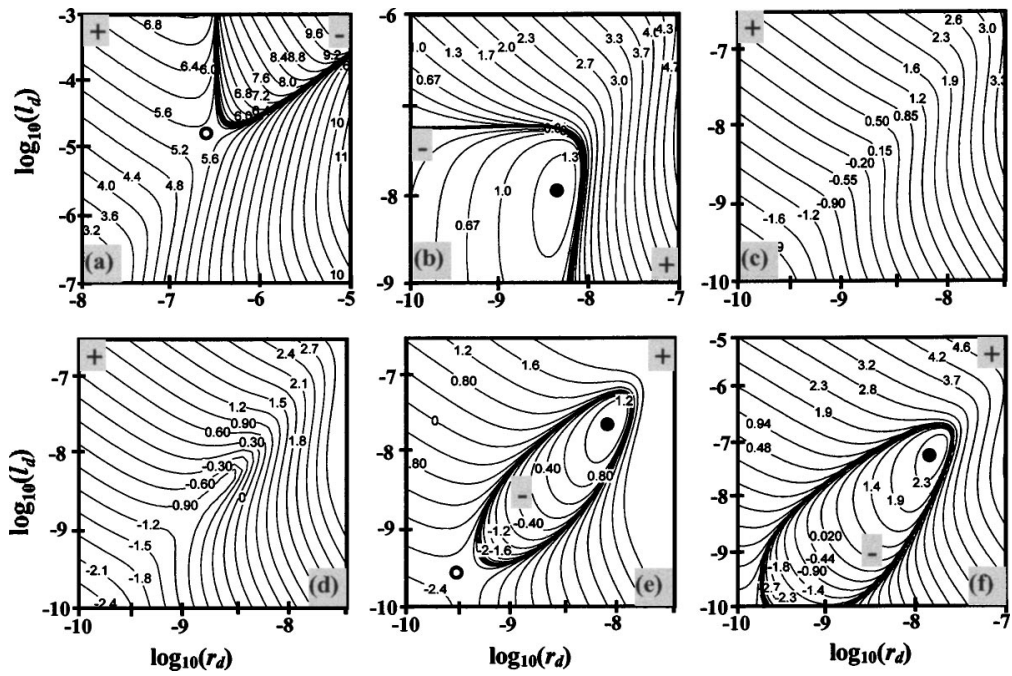

FIG. 3. The free energy surface for domain switching in: (a) a uniform field and the field produced by a point charge (b) on the surface and (c), (d), (e) above the surface. (f) The free energy surface for a realistic tip shape. Plotted is the logarithm of the absolute value of the energy in eV. Solid lines separate regions of opposite signs, indicated by the plus and minus signs. Saddle points $(\bigcirc)$ and local minima $(\bullet)$ are shown. case. Instead, the tip per se acts as a nucleation center.

To examine this behavior quantitatively, the change in free energy due to the presence of the tip is calculated. The electroelastic field distribution generated by the PFM tip is highly nonuniform and the corresponding domain free energy is

$$
\Delta G_{\text {bulk }}=\int_{V} \Delta g_{\text {bulk }}(\mathbf{r}) d V=2 \pi \int_{0}^{l_{d}} d z \int_{0}^{r(z)} \Delta g_{\text {bulk }}(r, z) r d r,
$$

where $r(z)=r_{d} \sqrt{1-z^{2} / l_{d}^{2}}$. An initial insight into the PFM switching phenomena can be obtained using point charge models that are applicable if domain sizes $l_{d}, r_{d} \gg R, a$, where $R$ is the tip radius and $a$ is the contact radius [Fig. 2(b)], and provided that the singularity at the origin is weak enough to ensure convergence of the integral in Eq. (4). For ferroelectric switching induced by a point charge $q_{s}$ located on the surface, the integral in Eq. (4) can be taken analytically and $\Delta G_{\text {bulk }}=d r_{d} l_{d} /\left(l_{d}+\gamma r_{d}\right)$, where $d=2 P_{s} q_{s} /\left(\varepsilon_{0}+\sqrt{\varepsilon_{11} \varepsilon_{33}}\right)$ and $\gamma=\sqrt{\varepsilon_{33} / \varepsilon_{11}}$. The free energy surface for $q_{s}=100 e^{-}$is shown in Fig. 3(b).

The tip-induced domain switching can be compared to the Landau theory of phase transitions, with domain size as an order parameter. In the case of a point charge on the surface, domain formation is a second order phase transition, since in the vicinity of the point charge, the electrostatic field is infinitely large and nucleation always occurs [scenario II in Fig. 1(b)]. Similar behavior is expected for a point charge inside a ferroelectric material.

This situation changes drastically for a point charge above, rather than on, the ferroelectric surface. In this case, Eq. (4) can also be evaluated analytically; however, the corresponding closed-form solution is rather cumbersome. For a point charge $q_{a}$ located at height $h$ above the surface, the field in the ferroelectric is finite and nucleation is a first order phase transition. Free energy surfaces for several point charge magnitudes and $h=10 \mathrm{~nm}$ are shown in Figs. 3(c)-3(e). For $q_{a}=100 e^{-}$, the free energy is positive for all $l_{d}, r_{d}$ and a domain does not form, corresponding to scenario I in Fig. 1(b). For $q_{a}=200 e^{-}$the free energy surface develops a kink. Finally, for $q_{a}=400 e^{-}$the free energy minimum corresponding to a stable domain and a saddle point corresponding to the activation energy for nucleation are clearly seen, corresponding to scenario III in Fig. 1(b). The characteristic domain size is on the order of several unit cell parameters, suggesting that the applicability of continuum theory for the description of the nucleation step can be limited. The behavior in Fig. 3 closely resembles the free energy surface evolution in a first-order phase transition. For large charge magnitudes or small charge-surface separations, the activation energy becomes small, and the free energy surface resembles that for a point charge on the surface, corresponding to scenario III in Fig. 1(b).

\section{SWITCHING IN THE WEAK INDENTATION REGIME}

This analysis can be extended to spherical tip geometry by modeling the tip by a distribution of image charges. For the weak indentation regime (contact radius $a=0$ ), the field distribution can be derived using the image charge method. ${ }^{15,22,23}$ The charge distribution in the tip is represented by the set of image charges $Q_{i}$ located at distances $r_{i}$ from the center of the sphere such that

$$
\begin{aligned}
& Q_{i+1}=\frac{\kappa-1}{\kappa+1} \frac{R}{2(R+d)-r_{i}} Q_{i}, \\
& r_{i+1}=\frac{R^{2}}{2(R+d)-r_{i}},
\end{aligned}
$$

where $R$ is the tip radius, $d$ is the tip-surface separation, $Q_{0}=4 \pi \varepsilon_{0} R V, r_{0}=0$, and $V$ is the tip bias. The tip-surface capacitance is $C_{d}(d, \kappa) V=\sum_{i=0}^{\infty} Q_{i}$ and for small tip-surface separation $C_{d}(\kappa)=4 \pi \varepsilon_{0} R[(\kappa+1) / \kappa-1] \ln [(\kappa+1) / 2] .{ }^{24}$ The potential distribution inside the dielectric material induced by charge $Q_{i}$, at distance $R+d-r_{i}$ above the surface is ${ }^{25}$

$$
V_{i}(\rho, z)=\frac{Q_{i}}{2 \pi \varepsilon_{0}(\kappa+1)} \frac{1}{\sqrt{\rho^{2}+\left(r_{i}+z / \gamma-d-R\right)^{2}}},
$$

where $\gamma=\sqrt{\kappa_{33} / \kappa_{11}}$ and $\rho$ is the radial coordinate on the surface. The total potential inside the ferroelectric is 


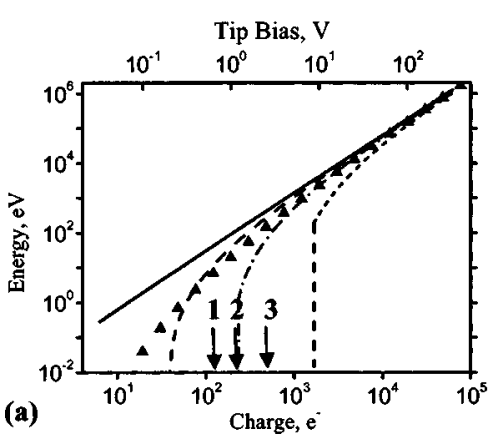

$$
V_{i c}(\rho, z)=\sum_{i=0}^{\infty} V_{i}
$$

At points that are far from the contact area, $\rho, z \gg R$, the potential distribution is similar to that generated by a point charge $Q=C_{d} V$ on the anisotropic dielectric surface

$$
V_{i c}(\rho, z)=\frac{C_{d} V}{2 \pi \varepsilon_{0}(\kappa+1)} \frac{1}{\sqrt{\rho^{2}+(z / \gamma)^{2}}} .
$$

An approximation of a point charge $q_{a}=C_{d} V$ located at distance $R$ from the surface was used in Ref. 9 to describe the domain switching processes for a domain size larger than the tip radius. While valid for $r_{d}, l_{d}>R$, the point-charge approximation is no longer valid for small separations from the contact area and a full description using Eqs. (5) and (7) is required. The crossover from sphere-plane to the asymptotic point charge behavior occurs at distances comparable to the tip radius. Given the characteristic size of the tip (on the order of 10-200 nm), a rigorous description of the domain nucleation and early stages of domain growth in the weak indentation limit necessitates the use of Eq. (7). This is also the case for applications such as ferroelectric domain patterning in thin films, in which the minimal experimentally achievable domain size (radius $\sim 20 \mathrm{~nm})^{5}$ is comparable to the tip radius of the curvature.

The free energy surface calculated using Eqs. (6) and (7) for a tip radius $R=50 \mathrm{~nm}$ and bias $V=5 \mathrm{~V}$ is shown in Fig. 3(f). Similar to the case with a point charge above the surface, domain nucleation is possible only above a certain threshold value of tip bias, corresponding to a first-order phase transition. The bias dependence of the domain energy [corresponding to the minimum in Figs. 3(b), 3(e), and 3(f)] and the equilibrium lateral domain size $r_{d}$ for $\mathrm{BaTiO}_{3}$ in a point charge model for different charge-surface separations (assuming the spherical tip geometry) are shown in Figs. 4(a) and 4(b). Notably, behavior for a spherical tip resembles that for a point charge at $\sim 1 \mathrm{~nm}$ separation. This indicates that tip-induced switching cannot be adequately described by a point charge located at the center of the curvature of the tip, due to the concentration of image charges at the tip-surface junction as expected for a high- $k$ material. The effective charge-surface separation and hence the critical nucleation bias will be larger for materials with lower dielectric constant such as $\mathrm{LiNbO}_{3}$. The domain size and energy plotted as functions of tip charge $Q_{\text {tip }}=C_{d} V_{\text {tip }}$ are almost independent of the effective tip radius (not shown), suggesting that the tip charge is the parameter that controls the mechanism of the switching process.

For large domain sizes, $r_{d}, l_{d}>a$, the point charge approach can also be applied in the strong indentation case, when the capacitance of the tip-surface contact area $C_{\mathrm{ca}}$ $=4 \kappa \varepsilon_{0} a$ is larger than that of the spherical part of the tip $C_{\mathrm{ca}}>C_{d \cdot}{ }^{15}$ In this case, the contact area capacitance must be used in Eq. (8). In the general case of piezoelectric indentation, when both tip bias and indentation force contribute to the tip charge through dielectric and inverse piezoelectric coupling, the point charge model can be applied using an effective tip charge determined through the stiffness relation for the appropriate indenter geometry [e.g., Eq. (24) in Ref. 15 for a spherical tip].

\section{SWITCHING IN A STRONG INDENTATION REGIME}

To extend this analysis of the ferroelectric polarization switching processes to a realistic tip geometry, including the effect of contact area $(a>0)$ and elastic stress $(P>0)$ and to describe nucleation and the early stages of domain growth, we develop a model based on the exact solutions for the electroelastic field structure for the strong indentation case. ${ }^{15}$

The total potential induced by the tip can be represented as a sum of the electrostatic contribution due to the tip bias, and the electromechanical contribution due to the load force, and the electromechanical coupling in the material as $\psi$ $=\psi_{\mathrm{el}}+\psi_{\mathrm{em}}$. For tip biases on the order of several Volts and larger typically used in the PFM experiments, $\psi_{\mathrm{el}} \gg \psi_{\mathrm{em}}$ and the electrostatic potential under the tip is

$$
\psi_{\mathrm{el}}=-\frac{2 \psi_{0} H^{*}}{\pi} \sum_{j=1}^{3} \frac{k_{j}^{*}}{\gamma_{j}^{*}}\left(N_{j}^{*} C_{3}^{*}+L_{j}^{*} C_{4}^{*}\right) \arcsin \left(\frac{a}{l_{2 j}}\right),
$$

where the relevant constants are defined in Ref. 15. Equation (9) takes into account electromechanical coupling and is a generalization of the electrostatic model for a conducting disk on an anisotropic dielectric half-plane for the case of a transversely isotropic piezoelectric material.

The analysis of the switching process is performed for $\mathrm{BaTiO}_{3}$, with $R=50 \mathrm{~nm}$ and $a=3 \mathrm{~nm}$, and an indentation force of $P=92 \mathrm{nN}$. To quantify the domain switching behavior, the free energy density Eq. (1) is calculated using an exact formula for the electrostatic field, while the bulk contribution to the free energy Eq. (4) and the minimum of the domain free energy as a function of $l_{d}, r_{d}$ are calculated numerically. ${ }^{26}$ The bias dependence of the domain energy 

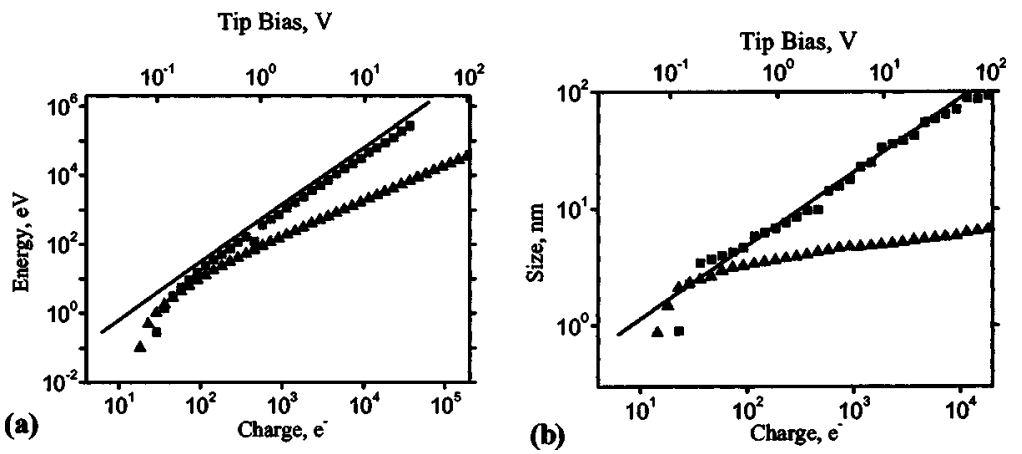

FIG. 5. The domain energy (a) and lateral domain size (b) as a function of effective tip charge and tip bias for a point charge (solid), ferroelectric switching (ם) and ferroelectroelastic switching $(\mathbf{\Lambda})$ for $\mathrm{BaTiO}_{3}$, with $R$ $=50 \mathrm{~nm}$ and $a=3 \mathrm{~nm}$, and an indentation force of $P$ $=92 \mathrm{nN}$.

and lateral domain size is illustrated in Figs. 4(a) and 4(b), respectively. Note that for large biases, the switching behavior is well approximated by the point charge model where the charge magnitude is now related to the indentation parameters by the stiffness relation

$$
Q=\frac{4 a^{3} C_{2}^{*}}{3 \pi R}+\frac{2 a \psi_{0} C_{4}^{*}}{\pi},
$$

where $C_{2}^{*}=15.40 \mathrm{~N} / \mathrm{V} \mathrm{m}$ and $C_{4}^{*}=48.54 \times 10^{-9} \mathrm{C} / \mathrm{mV}$ for $\mathrm{BaTiO}_{3}$. This is an extension of the point charge model that takes into account the electromechanical coupling in the material.

Tip induced switching behavior in the strong indentation regime is qualitatively similar to that corresponding to a point charge above a ferroelectric surface. Domains can nucleate only above a critical voltage. For $\mathrm{BaTiO}_{3}$, this minimum is calculated to be $V>0.1 \mathrm{~V}$, with the minimum domain size being $\sim 3 \mathrm{~nm}$, i.e., comparable to the contact radius, in agreement with an early suggestion by Gruverman. ${ }^{27}$ Tip flattening during imaging will result in an increase of the contact radius and hence will increase the minimum achievable domain size. In contrast, for a small contact area, switching is dominated by the field produced by the spherical and conical parts of the tip, resulting in optimal conditions for domain nucleation.

The applicability of the point charge model is limited to the first-order ferroelectric and ferroelastic switching processes. In particular, the integral in Eq. (4) converges only for $\alpha<2$, where $\alpha$ describes the asymptotic behavior for potential and strain in the form $f=x^{-\alpha}$, where $x$ is the distance from the tip-surface contact. For high order ferroelectric switching, both electrostatic and strain fields decay as $1 / x^{2}$; hence $\alpha=2$ for ferrobielectric, ferrobielastic, and fer- roelastoelectric switching and the integral [Eq. (4)] does not converge, necessitating the exact structure of the field to be taken into account.

To extend this analysis to ferroelectroelastic switching described by the second term in Eq. (1), the stress distribution inside the material is found as a solution of the mechanical indentation problem:

$$
\begin{aligned}
\sigma_{z z}= & \frac{4 H^{*}}{\pi R} \sum_{j=1}^{3} \alpha_{j}^{*}\left(N_{j}^{*} C_{1}^{*}+L_{j}^{*} C_{2}^{*}\right) \\
& \times\left\lfloor z_{j} \arcsin \left(\frac{l_{1 j}}{\rho}\right)-\left(a^{2}-l_{1 j}^{2}\right)^{1 / 2}\right\rfloor,
\end{aligned}
$$

where the constants are defined in Ref. 15. The free energy density for the ferroelectroelastic switching process $\Delta g_{\text {bulk }}$ $=-2 d_{33} E_{z} \sigma_{z z}$ is calculated numerically from Eqs. (9) and (11). Similarly to ferroelectric switching, the domain energy is calculated by Eq. (4) and numerical minimization yields domain size and energy.

The calculated bias dependence of the domain energy and the equilibrium lateral domain size for ferroelectroelastic switching are shown in Figs. 5(a) and 5(b), respectively. Similarly to ferroelectric switching, domain nucleation occurs only above a critical bias. At the same time, the domain size is a much weaker function of the bias, reflecting a faster decay of the electroelastic fields, as the distance from the tip-surface junction increases.

Examples of the ferroelectroelastic switching in PFM are illustrated in experiments using a single crystal of lithium niobate (LN) and also a thin film of PZT in Fig. 6. In these experiments, a single voltage pulse was applied via the tip to a ferroelectric sample (with the tip held under a controlled
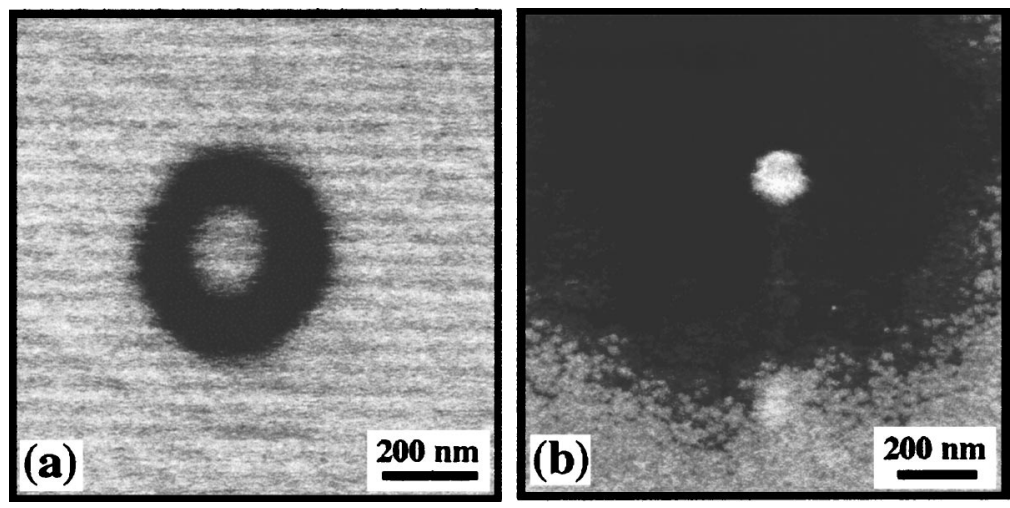

FIG. 6. Examples of ferroelectroelastic domain switching in: (a) a lithium niobate single crystal and (b) a PZT thin film. Tip bias is $-200 \mathrm{~V}$ in (a) and $-6 \mathrm{~V}$ in (b). Domains generated as a result of ferroelectric and ferroelectroelastic switching (dark and bright regions, respectively) exhibit dramatically different sizes. 
loading force) followed by imaging of the resulting domain structure by PFM. In both images, a dark region corresponds to a ferroelectrically switched domain. In addition, an inverse domain (with polarization opposite to the applied field) can be seen as a white spot in the center of the larger dark region. This bright region is a domain generated due to ferroelectroelastic switching as a result of combined action of mechanical stress and electric field. ${ }^{13}$ Note that the ferroelectroelastically switched domains are significantly smaller than ferroelectric domains, in agreement with predictions in Fig. 5(b). The fact that the ferroelectroelastic switching has been observed in such different systems as perovskite thin film (PZT) and uniaxial single crystal (LN) infers a universal nature of this phenomenon.

\section{SUMMARY}

To summarize, the tip-induced nanoscale ferroelectric switching in the weak and strong indentation limits is analyzed using exact closed form solutions for the electroelastic fields. It is shown that domain nucleation can be described in terms of the Landau theory of phase transitions, where domain size is an order parameter and the applied bias plays the role of temperature. For a point charge on the surface or inside the ferroelectric, ferroelectric nucleation can be considered as a second order phase transition, while for a charge above the surface and for a realistic tip shape, the switching is of first order. In ferroelectric switching, the domain size is independent of the contact area and is determined solely by the tip charge or force. In contrast, in the high order ferroelectroelastic switching, the tip-surface contact contribution to the domain free energy dominates due to the much higher decay rate of the product of electrostatic and elastic fields. Similarly, domain nucleation and early stages of domain growth are sensitive to the exact structure of the electroelastic fields inside the material, necessitating further analysis of these phenomena for high density ferroelectric applications.

\section{ACKNOWLEDGMENTS}

Research was performed as a Eugene P. Wigner Fellow and staff member at the Oak Ridge National Laboratory, managed by UT-Battelle, LLC, for the U.S. Department of Energy under Contract No. DE-AC05-00OR22725 (S.V.K.). S.V.K. and A.P.B. acknowledge ORNL SEED funding. A.G. acknowledges financial support of the National Science Foundation (Grant No. DMR02-35632) and Bilateral U.S.Israel Science Foundation. The authors thank Professor E. Ward Plummer (University of Tennessee and ORNL) for valuable discussions.

${ }^{1}$ Nanoscale Characterization of Ferroelectric Materials, edited by M. Alexe and A. Gruverman (Springer, New York, 2004).

${ }^{2}$ Nanoscale Phenomena in Ferroelectric Thin Films, edited by S. Hong (Kluwer Academic, Boston, 2004).

${ }^{3}$ T. Morita and Y. Cho, Appl. Phys. Lett. 84, 257 (2004).

${ }^{4}$ H. Shin, S. Hong, J. Moon, and J. U. Jeon, Ultramicroscopy 91, 103 (2002).

${ }^{5}$ T. Tybell, P. Paruch, T. Giamarchi, and J.-M. Triscone, Phys. Rev. Lett. 89, 097601 (2002).

${ }^{6}$ K. Terabe, M. Nakamura, S. Takekawa, K. Kitamura, S. Higuchi, Y. Gotoh, and Y. Cho, Appl. Phys. Lett. 82, 433 (2003).

${ }^{7}$ S. V. Kalinin, D. A. Bonnell, T. Alvarez, X. Lei, Z. Hu, J. H. Ferris, Q. Zhang, and S. Dunn, Nano Lett. 2, 589 (2002).

${ }^{8}$ S. V. Kalinin, D. A. Bonnell, T. Alvarez, X. Lei, Z. Hu, R. Shao, and J. H. Ferris, Adv. Mater. (Weinheim, Ger.) 16, 795 (2004).

${ }^{9}$ M. Molotskii, J. Appl. Phys. 93, 6234 (2003).

${ }^{10}$ M. Molotskii, A. Agronin, P. Urenski, M. Shvebelman, G. Rosenman, and Y. Rosenwaks, Phys. Rev. Lett. 90, 107601 (2003).

${ }^{11}$ M. Abplanalp, PhD Natural Science thesis, Swiss Federal Institute of Technology, Zurich, 2001.

${ }^{12}$ A. L. Kholkin, V. V. Shvartsman, A. Yu. Emelyanov, R. Poyato, M. L. Calzada, and L. Pardo, Appl. Phys. Lett. 82, 2127 (2002).

${ }^{13}$ M. Abplanalp, J. Fousek, and P. Gunter, Phys. Rev. Lett. 86, 5799 (2001).

${ }^{14}$ M. Labardi, C. Polop, V. Likodimos, L. Pardi, M. Allegrini, E. Vasco, and C. Zaldo, Appl. Phys. Lett. 83, 2028 (2003).

${ }^{15}$ S. V. Kalinin, E. Karapetian, and M. Kachanov, Phys. Rev. B 70, 184101 (2004); cond-mat/0408223

${ }^{16}$ S. V. Kalinin and D. A. Bonnell, Phys. Rev. B 65, 125408 (2002).

${ }^{17}$ M. E. Lines and A. M. Glass, Principles and Applications of Ferroelectric and Related Materials (Clarendon, Oxford, 1977).

${ }^{18}$ G. A. Smolenskii, V. A. Bokov, V. A. Isupov, N. N. Krainik, R. E. Pasynkov, and A. I. Sokolov, Ferroelectrics and Related Materials (Gordon and Breach, New York, 1984).

${ }^{19}$ R. Landauer, J. Appl. Phys. 28, 227 (1957).

${ }^{20} \mathrm{Eq}$. (3) is valid only for $l_{d}>r_{d}$. To avoid this limitation, used here was the expression for the demagnetization factor for prolate ellipsoid from J. A. Osborn, Phys. Rev. 67, 351 (1945).

${ }^{21}$ F. Jona and G. Shirane, Ferroelectric Crystals (Dover, New York, 1993).

${ }^{22}$ J. D. Jackson, Classical Electrodynamics (Wiley, New York, 1998).

${ }^{23} \mathrm{M}$. Cohen (private communication).

${ }^{24}$ W. R. Smythe, Static and Dynamic Electricity (McGraw-Hill, New York, 1968).

${ }^{25}$ E. J. Mele, Am. J. Phys. 69, 557 (2001).

${ }^{26}$ Mathematica 5.0, Wolfram Research, Champaign, IL

${ }^{27}$ A. Gruverman, J. Hatano, and H. Tokumoto, Jpn. J. Appl. Phys., Part 1 36, 2207 (1997). 